\title{
FRACTAL APPROACH TO FORMING OF MODIFIED BIFURCATION DIAGRAMS IN PRACTICAL APPLICATIONS
}

\author{
Yury Kolokolov, Anna Monovskaya \\ Russian Research Institute of Hydrometeorological Information - World Data Center, \\ 6, Korolev str., 249035, Obninsk, Russia \\ 2kolo@mail.ru,www.meteo.ru
}

\begin{abstract}
The points connected with both improving the methods based on the use of the bifurcation analysis and opening new possibilities to use the bifurcation analysis in practical applications are discussed in the paper. So, the fractal approach to form modified bifurcation diagrams is developed. The approach is directed towards extending knowledge about the nonlinear dynamics state-of-the-art by means of the growth in the capacity and adequacy of information about nonlinear dynamics regularities and uncertainties. In particular, it is summarized the peculiarities of the fractal approach; it is signed the main ways to practical applications of the fractal approach - forecasting nonlinear dynamics, estimating the stability margin and risk.
\end{abstract}

Keywords: Bifurcation analysis, Uncertainties, Regularities, Practical applications, Forecasting, Stability margin, Risk estimating.

\section{INTRODUCTION}

The great importance of a bifurcation diagram is caused by the fundamental idea - to represent the inhere property of Nature connected with the regularities during the evolution of a nonlinear system. Taking into account this idea, the bifurcation analysis is widely ised for scientific researches of nonlinear dynamics regarding different kinds of systems, for example: researching into dynamical processes keeping under observation in power $[1,2]$, control [2, 3] and information [4,5] parts of technical systems; researching into dynamical processes keeping under observation in biological [6] and natural $[7,8]$ systems; revealing the evolution processes in social systems [9]; and so on. However, the restrictions concerning the use of bifurcation analysis results for practical applications remain yet quite hard. Such restrictions can be conditionally divided in two groups.

The first group of the restrictions follows from unavoidable uncertainties, which appear in the nonlinear system dynamics during intricate interrelations between several nonlinear phenomena under noise effects. As a result, there is the problem of building the mathematical model characterized by the both properties: the adequacy regarding the completeness of nonlinear phenomena variety; the adequacy regarding the accuracy of determining the bifurcation boundary allocations. The first group restrictions are mainly softened by adjusting the parameters of a system by the trial-and-error method $[2,10]$. In this case, at first, computer calculations are carried out for a simplified mathematical model of the system with the purpose to determine a preliminary solution. Then, experimental researches are carried out to make more precise the computer solution. But, the difference between a computer simulated bifurcation diagram and an experimental bifurcation diagram can remain essential even after mathematical model complications [2]. It leads to the necessity to provide for great stability margins while design a control system using in a responsible technological process.

The second group of the restrictions follows from both multidimensional phase and multidimensional parametrical spaces, which are involved in imaging the evolution of nonlinear dynamics. As a result, there is the problem of informational visualization, because a bifurcation diagram is either not originally designed to show or can partly show some phenomena and some characteristics which are necessary to analyse. For example, the intermittency is shown with distortions, both the ripple amplitude and the average value of an analyzed phase variable are partly shown, a symbolic characteristic of a periodical process is not shown, and so on [11]. The second group restrictions are mainly softened by using the modifications of bifurcation diagrams. The simplest variant of such modification is to exhibit 
the evolution of some necessary characteristic of a periodic process (for example, the symbolic characteristic) similar to the evolution of a phase variable $[11,12]$. Other example, it can be forming of the fractal structures, which allow to connect uniquely in a special 2D-projection about five or six coordinates of phase and parametrical spaces [13].

The mentioned problems of the mathematical model adequacy and the informational visualization have a deep interrelation. The interrelation is stipulated not only by uncertainties and regularities of nonlinear dynamics evolution, but also by possibilities and restrictions of the search tools used. This problem statement is considered in the fractal approach to forming of modified bifurcation diagrams. The basic idea of the fractal approach is to pick up such viewpoint on nonlinear dynamics evolution that this viewpoint is the most proper for solving the certain scientific or practical task. As a result, it becomes possible to improve the methods based on use of the bifurcation analysis and to open new possibilities to use the bifurcation analysis in practical applications. In the paper the peculiarities of the fractal approach to forming of modified bifurcation diagrams are summarized. Also it signs the main ways to practical applications of the fractal approach - forecasting of nonlinear dynamics, estimating the stability margin and risk. The results presented in the paper are got by experimental researches.

\section{EXPERIMENTAL SETUP}

Aspiration to practical applications of the bifurcation analysis leads to focus attention on experimental researches. The benefit of the experimental way is connected with the presentation of nonlinear dynamics evolution without destructions and distortions owing to the intricate interrelations between nonlinear phenomena and noise in contrast to the computational way based on use of mathematical models with unavoidable simplifications. The results of the experimental researches presented in the paper were made on the experimental setup "DC-DC buck converter" $(60 \mathrm{~W})$, the functional diagram of which is presented in Fig. 1a. The experimental setup was desined by the modular approach taking into account the widely-used schematic circuits (a PWM-regulator, a buck converter, a power supply, and so on). The information-measuring system was desined by analogy with the experimental setup "DC-DC drive" $(1 \mathrm{~kW}) \quad[2,11,14]$. In particular, the digital oscilloscope Tektronix TDS3014B is used for interactive signal imaging (channel " 1 "), first of all, for the synchronized imaging of the current (i) and voltage in the buck converter power circuit, and also the synchronizing function voltage $\left(U_{C F}\right)$ and the switching function voltage $\left(U_{K F}\right)$. Time series is acquired by the data acquisition system E20-10 produced by L-Card company (Moscow, Russia), which is physically the extension board connected to the PC via USB port $[11,14]$. The system consists of a 14-bit analog-to-digital converter (ADC) and a direct memory access controller providing the continuous data piping to the PC for posterior processing (channel " 2 "). Additionally, the specialized real-time channel (channel " 3 ") is realized through the data processing and data transferring subsystems. The data processing subsystem includes the RISC-microcontroller (ARM7, NXP/Philips). The data transferring subsystem includes the erasable programmable logic device (Cyclone I, ALTERA) and the interface translator (FT245RL, FTDI). The channel " 3 " is used for realization and investigation of real-time identification, forecasting and adaptation algorithms based on time series fragmentation [11, 14].

The algorithm of bifurcation diagram building is well-known and is based on the use of Poincaré's periodicity condition. Its data acquisition cycle consists of the following procedures: setting the actual parameters; waiting the running transient completion; acquiring the phase variable samples with the synchronizing frequency $(1 / T)$; data saving. The cycle is repeated with variation of each parameter with the specified step and direction within the specified range. Certainly, an experimental setup can not guaranty any initial condition for each phase variable, any range and any step for each variable parameter, any disturbance at each moment, and so on. Nevertheless, if it is known both regularities and uncertanties of the considered system nonlinear dynamics, then it can be proposed variants "to detour" such experimental limits. The more regularities and uncertanties are known, the less limits there are. Taking into account this rule, it is accepted the following peculiarity of data asquicition in relation to the abovementioned algorithm of bifurcation diagram building: the sampling frequency is higher in several tens; the sampling duration is longer in several times. For example, an experimental bifurcation diagram presented in Fig.1b exhibits the evolution of nonlinear dynamics through the period doubling 1-24-.. scenario. The fragments of time series around Fig. 1b illustrate the periodic processes mapped by the bifurcation diagram branches (from left to right: the 1-process; the 2-process; intermittency of the 1and 2-processes). 

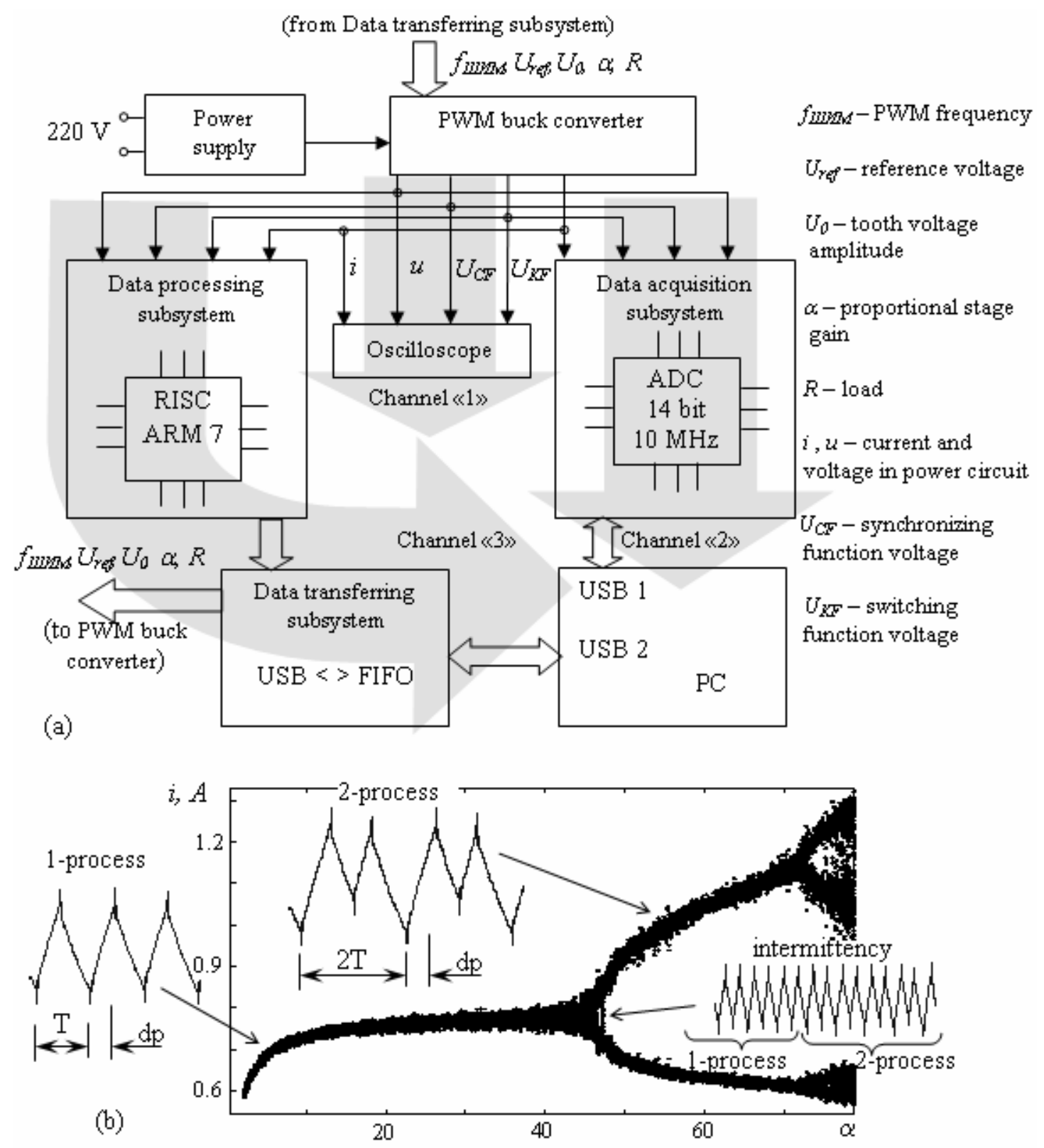

Fig. 1 - The functional diagram of the experimental setup (a); an example of the bifurcation diagram, R-value is equal to $10 \Omega(b)$

\section{MODIFICATIONS OF BIFURCATION DIAGRAMS AND REGULARITIES}

The modified bifurcation diagram represents a projection that can be built by use of the same set of time series. Modifications exhibit the possibility of different shapes in the case of the same bifurcation diagram. The shape depends on the choice of the moment of $i$-sampling regarding the $T$-period beginning (in other words, the choice of Poincare's mapping) and on potentialities of special operator transformations. All the modifications are regular. Let's build the dependence of $d m / d m_{\max }$ on $d p$ within the limits of periodic process existence, where $d m$ is the averaged distance between the 2-process branches, $d m_{\max }$ is the maximum $d m$-value, $d p$ is the relative duration from the $T$-period beginning to the moment of $i$-sampling in relation to the $T$-period duration (Fig. 1b). The range of the $d p$-values can be divided into two parts (Fig. 2a): from $d p=40 \%$ to $d p=71 \%$ there is $d m$-increasing; from $d p=71 \%$ to $d p=40 \%$ there is $d m$-decreasing with two bends near $d p=100 \%$ ( $T$-period beginning) and near $d p=20 \%$. This regularity remains similar with variation of parameters. Also, the fractal regularities of shifting the fixed points of mapping in the phase space will be observed [13]. For example, bifurcation diagrams will be regularly shifted downward along $i$-axis with $R$-value increasing (Fig. 2b). So, both the shape of the same bifurcation diagram and the disposition of a set of bifurcation diagrams in the phase space can be modified by the choice of Poincare's mapping. 

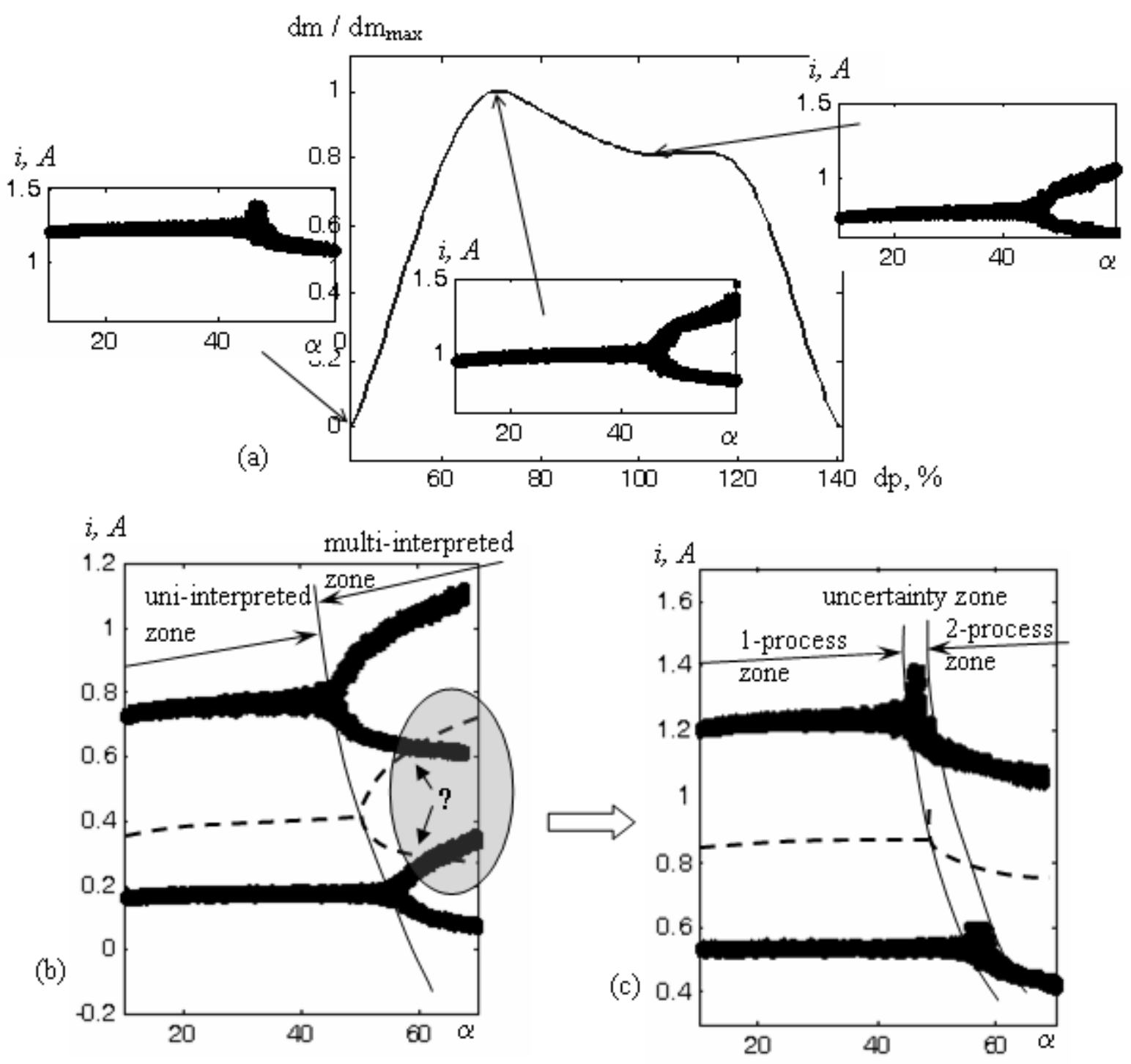

Fig. 2 - The dependence $d m / d m_{m a x}$ on $d p(a) ; B_{71}$-bifurcation diagrams (b) and $B_{40}$-bifurcation diagrams (c) with R-value equal to $10 \Omega$ and $30 \Omega$

This possibility is very important for the realization the fractal methods of real-time forecasting of nonlinear dynamics [13]. The main idea of the method is connected with forming of secondary, tertiary and so on fractal structures, which allow to connect uniquely in a special 2Dprojection about five or six coordinates of phase and parametrical spaces. To realize this connection a special operator transformation is used:

$$
X \stackrel{F_{V}}{\longrightarrow} \mathrm{V}
$$

where $X$ is a vector of phase variables in a multidimentional phase space, $F_{V}$ is a piece-wise operator, $V$ is a vector of images in a special 2Dprojection. The physical meaning of the transformation (1) is to eliminate the overlapping of branches of several bifurcation diagrams in the phase space. For example, from the Fig. $2 b$ it is necessary to eliminate either all the upper or all the bottom branches of the 2-process. Otherwise, the bifurcation diagram added between the uppermost and bottommost bifurcation diagrams will overlap one or several neighbour bifurcation diagrams (the dotted diagram in Fig. 2b).

The transformation by $F_{V}$ - operator can be quite laborious for realization. At the same time, it turns out that the same result can be got by using the $d m / d m_{\max }$-dependence on $d p$ as the choice of the $d p$ value with which $d m=0$. The bifurcation realization with the certain $d p$-value will be hereafter called the $B_{d p}$-bifurcation diagram («Branch»-bifurcation diagram), where the $d p$-index denotes conditions under which the diagram was built. The result of the corresponding modifications the bifurcation diagrams Fig. 2b is presented in Fig. 2c. The figure illustrates the existence of three zones: the 1-process zone; the 2-process zone; the narrow streak of an 

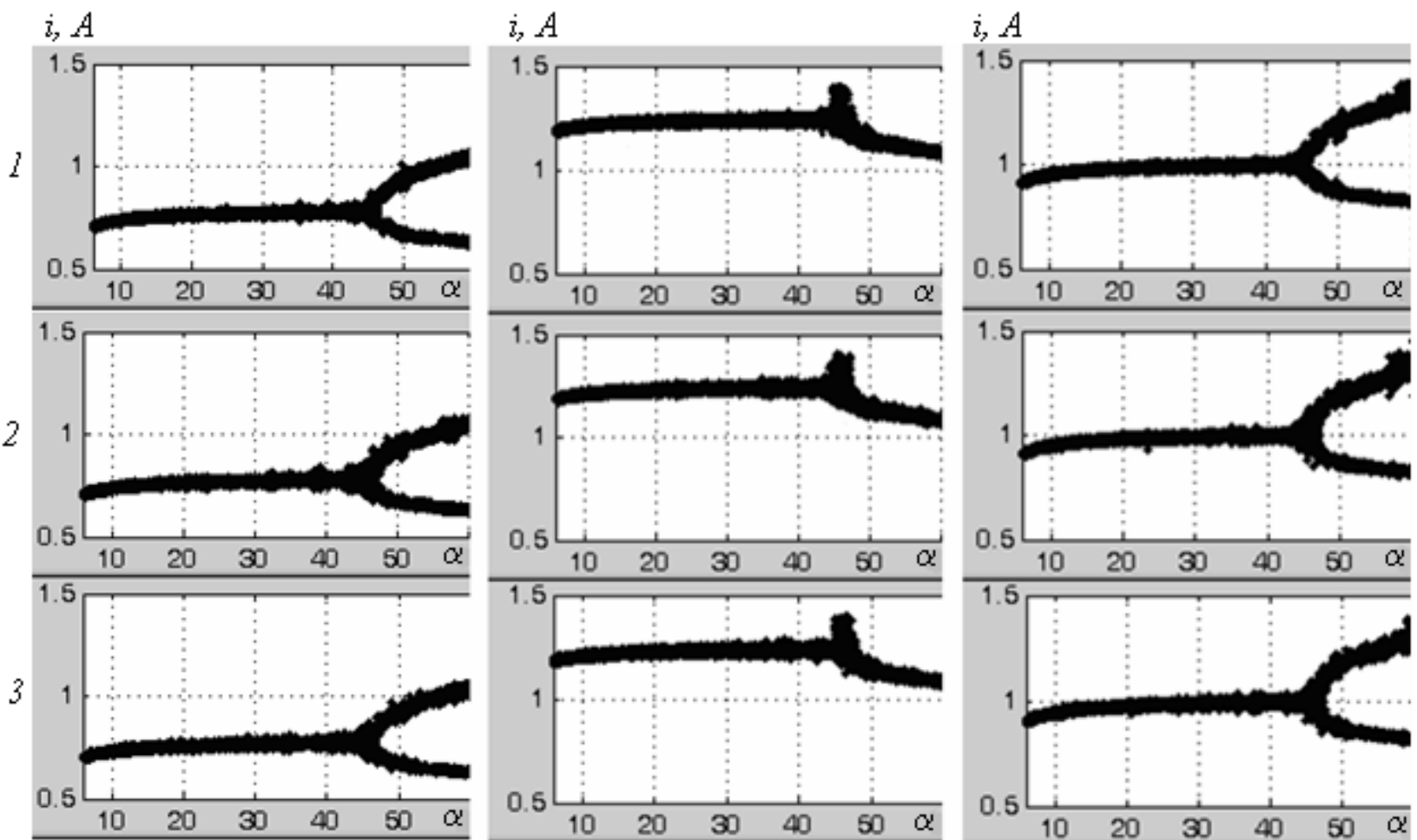

(a) $B_{0}$ - bifurcation diagrams

$B_{40}$ - bifurcation diagrams

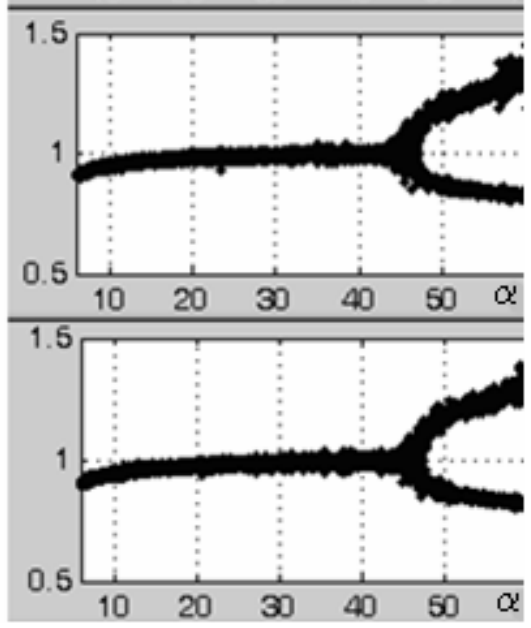

$B_{\tau_{1}}$ - bifurcation diagrams

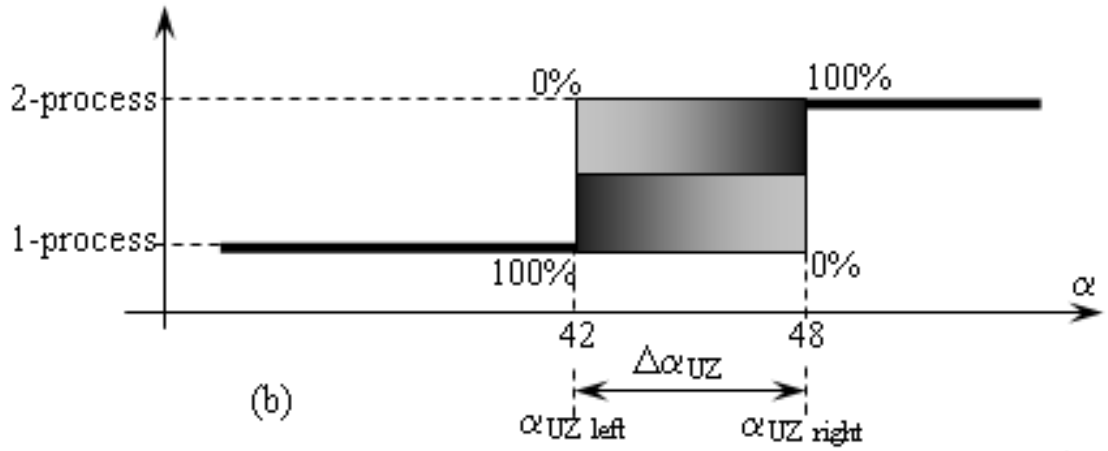

(c)

(d)

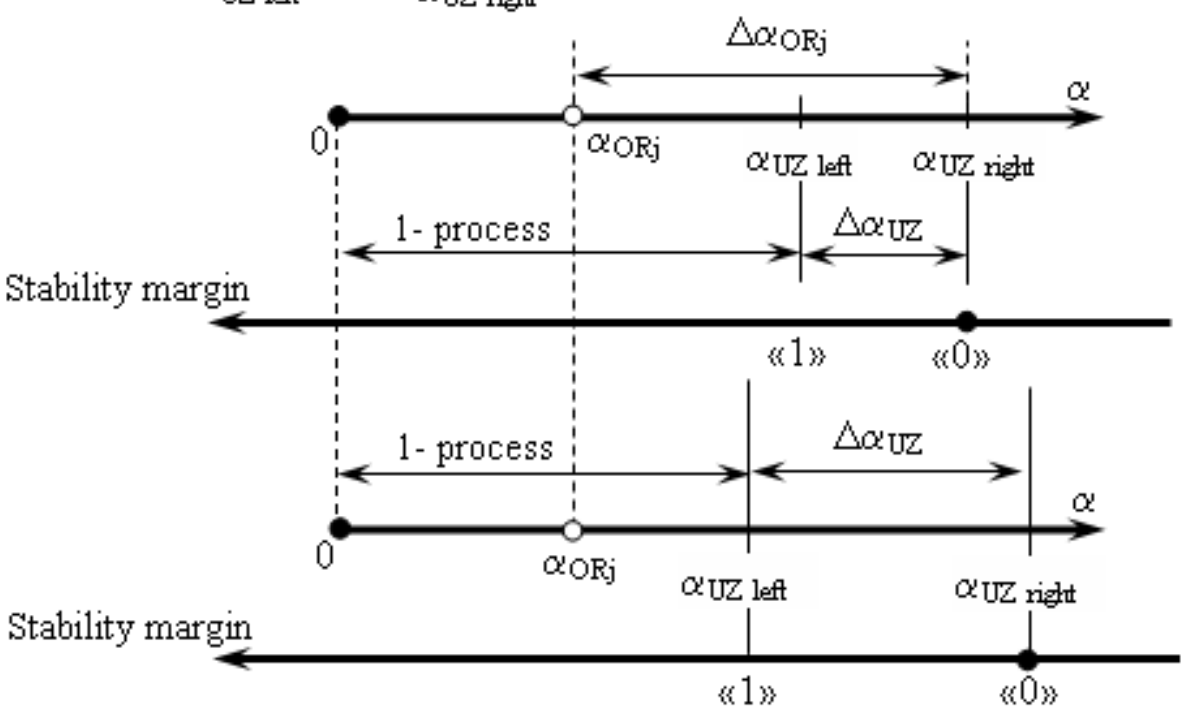

Fig. 3 - Three lines of the bifurcation realizations with R-value equal to $10 \Omega$, where three columns of $B_{0^{-}}, B_{40^{-}}$, $B_{71}$ - bifurcation diagrams are correspond to each bifurcation realization (a);

an example of $J$-bifurcation diagram (b); illustrations to the stability margin (c,d) 
uncertainty zone $\left(\Delta \alpha_{U Z}\right)$. Experimental researches show that the $\Delta \alpha_{\mathrm{UZ}}$-zone can not be eliminated because it is the particular property of any scenario of nonlinear dynamics evolution $[11,15]$. Since the transformation (1) is not originally designed to reveal the $\Delta \alpha_{\text {UZ }}$-zone then using the $B$-bifurcation diagrams allows to increase in adecuacy of the preliminary information for the fractal methods of real-time forecasting of nonlinear dynamics.

\section{REALIZATIONS OF BIFURCATION DIAGRAMS AND STABILITY MARGIN}

The unavoidable influence of "delicate" nonlinear phenomena (similar to a hysteresis, an intermittency and so on), noise and unknown parameters on a bifurcation point location leads to the conception of the bifurcation realization [11] the experimental bifurcation diagram built with the particular combination of internal and external parameters, and also with the particular direction and the particular step of the bifurcation parameter quasi-static variation. Processing a set of bifurcation realizations allows to get the generalized and averaged information. So called the $J$-bifurcation diagram ("Joint"- bifurcation diagram) is used to show this information [11]. In general case $J$ bifurcation diagrams allow to analyze either several kinds of the modifications for one bifurcation realization or the same kind of the modification for several bifurcation realizations. For example, let's consider three bifurcation realizations (three lines 1, 2, 3 in Fig. 3a) with three kinds of the $B$ bifurcation diagrams (three columns from left to right, accordingly: $d p=0 \%, d p=40 \%, d p=71 \%$ ). The figure shows the possibility of some differences while the bifurcation boundary is exibited in nine variants. The $\Delta \alpha_{\mathrm{UZ}}$-zone appears by combining these variants. In particular, here the $\Delta \alpha_{\mathrm{UZ}}$-zone range is equal about $15 \%$ of the 1 -process range that can be illustrated by one of the $J$-bifurcation diagrams (Fig. 3b). The 1-process probability is equal to $100 \%$

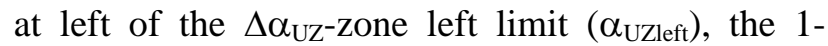
process probability is equal to $0 \%$ at right of the $\Delta \alpha_{U Z}$-zone right limit ( $\alpha_{\text {UZright }}$ ), a system behavior within the $\Delta \alpha_{\mathrm{UZ}}$-zone is uncertain. So it becomes possible to propose both the physical meaning and the principle of the quantitative assessment for the stability margin based on the nonlinear dynamics nature.

The commonly accepted meaning of the stability margin is connected with some nonzero distance between the actual $\left(\alpha_{\mathrm{ORj}}\right)$ and crucial values of a control system parameter, where the crucial value means the loss of the operating process stability. For example, if the 1-process is the operating and the 2- process is the abnormal, then it is necessary to answer two questions. First, where the bifurcation point locates? But taking into account system behavior within the $\Delta \alpha_{U Z}$-zone, the answer this question cannot be unique. Second, how long the distance between actual and crucial values should be? At present, this distance is determined in a wide range and is validated on the basis of the empirical considerations, for example $[16,17,18]$. Moreover, the operating process stability margin should correspond to the «worst combination» of parameters. But it is difficult to determine because of the changes over time in internal and external parameters in wide ranges, for example, $[1,2,10]$. Let's demonstrate that a physical meaning of the stability margin can approach the reality by the following assumption: the $\Delta \alpha_{\mathrm{Uz}}$-zone is the unit of measurement to estimate the stability margin.

In this case the stability margin $\left(\mathrm{S}_{\mathrm{ORj}}\right)$ is calculated by the way (Fig. 3c):

$$
\mathrm{S}_{\mathrm{ORj}}=\Delta \alpha_{\mathrm{ORj}} / \Delta \alpha_{\mathrm{UZ}}
$$

If $\alpha_{\mathrm{ORj}}$ belongs to the 1-process range, then $\mathrm{S}_{\mathrm{ORj}}>1$. By analogy, if $\alpha_{\mathrm{ORj}}$ belongs to the 2-process range, then $\mathrm{S}_{\mathrm{ORj}}<0$. The complication of operational conditions leads to widening the $\Delta \alpha_{\mathrm{UZ}}$-zone. For example, let the $\Delta \alpha_{U Z}-z o n e$ in Fig. $3 d$ is wider in comparison with the $\Delta \alpha_{\mathrm{UZ}}-\mathrm{zone}$ in Fig. 3c, and let the $\alpha_{\mathrm{ORj}}$-value is the same for the both cases. Then the $\mathrm{S}_{\mathrm{ORj}}$-value in Fig. $3 \mathrm{c}$ is greater than the $\mathrm{S}_{\mathrm{ORj}^{-}}$ value in Fig. $3 \mathrm{~d}$. The consequence from this property is concordant with the practical experience: the more various operating regimes there are, the more narrow the 1-process range is. Next, the minimal stability margin guaranteeing the $100 \%$ probability of the 1 process stability takes place with the left limit of the $\Delta \alpha_{\mathrm{UZ}}$-zone, where $\mathrm{S}_{\mathrm{ORj}}=1$. The decrease in this probability occurs from $\alpha_{\text {UZleft }}$ to $\alpha_{\text {UZright }}$. Correspondingly, the farther $\alpha_{\mathrm{ORj}}$ from the bifurcation boundary is, the greater $\mathrm{S}_{\mathrm{ORj}}$-value is. The probability of the 1-process stability disappears completely to $\alpha_{\text {Uzright, }}$ so, the stability margin becomes equal to zero.

\section{PIECE-WISE BIFURCATION DIAGRAMS AND RISK ESTIMATING}

At present, the risk estimating is based on the use of statistic methods. If one has in mind the risk estimating in the cases of qualitative leaps of system states, then it seems to be more valid to take into account the risk of bifurcation phenomena. Correspondingly, the use of only statistic methods becomes insufficiently complete owing to essential 

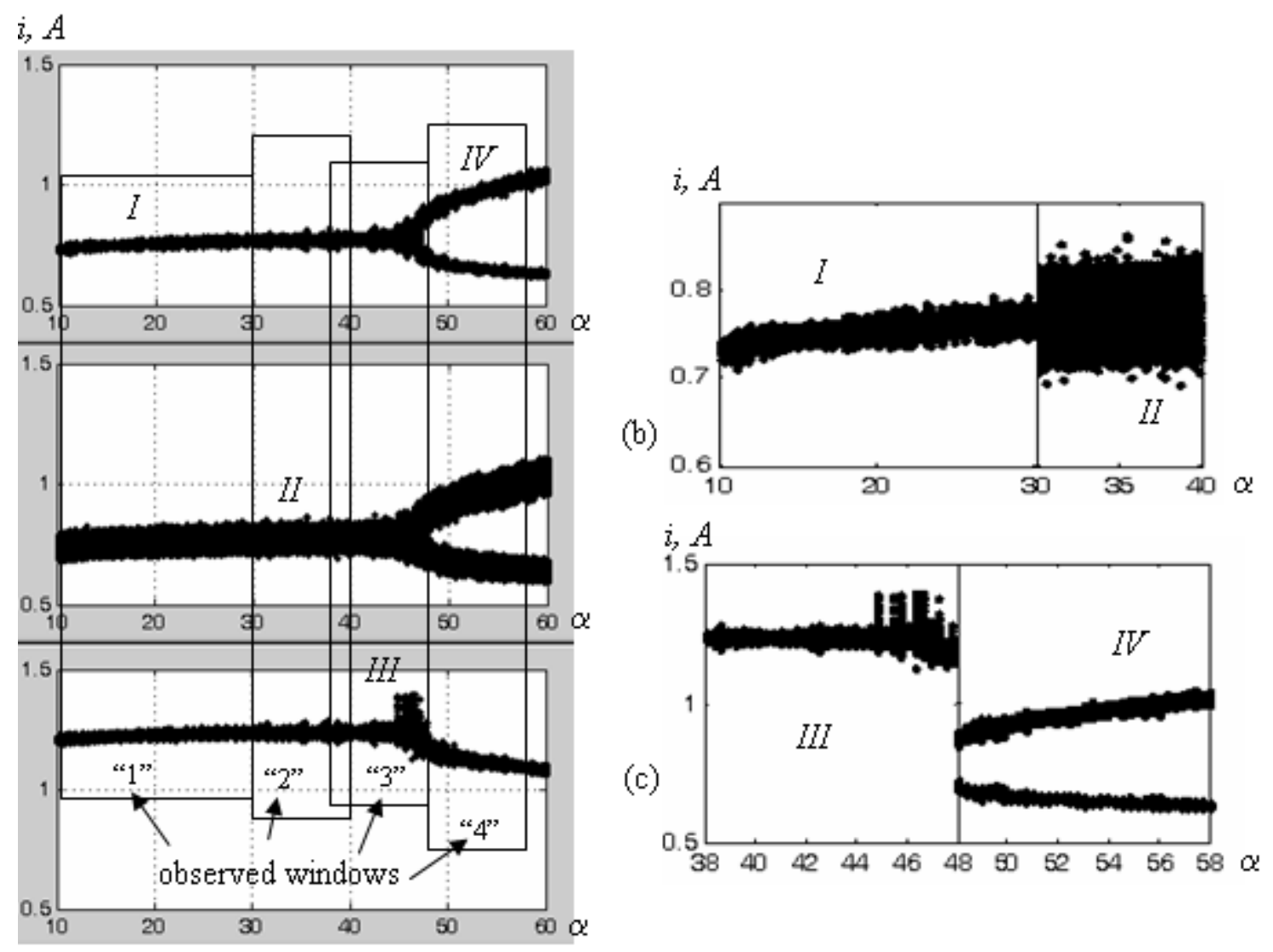

Fig. 4 - Three lines of $B_{0^{-}}, B_{40^{-}}, B_{71^{-}}$bifurcation diagrams for the same bifurcation realization with $R$-value equal to $10 \Omega$ (a); zoom-in combination of the fragments I and II, the observed windows " 1 " and " 2 " accordingly (b); zoom-in combination of the fragments III and IV, the observed windows “ 3 ” and "4” accordingly (c)

distinctions between the physical nature of uncertainty in accordance with statistic models and the physical nature of uncertainty in accordance with nonlinear models. Let's consider two examples to illustrate the problem statement (Fig. 4). In the left part of the Fig. 4 three modifications of the same bifurcation realization are presented (from up to bottom, accordingly: $B_{0}$-bifurcation diagram; $B_{2}$-bifurcation diagram; $B_{40}$-bifurcation diagram). All the diagrams are characterized by the blur of branches, where the range of noise is limited by the standard deviation $\pm 4 \sigma$ in relation to the preliminary calculated mathematical expectations for each branch. Let's assume that the prehystory of the system behavior is known within the range of $\alpha$ variation from 10 to 30 (the observable window " 1 " in Fig. 4a). Then the prospective system behavior within the range of $\alpha$-variation from 30 to 40 (the observable window "2" in Fig. 4a, the upper figure) will be concordant with the limits of statistical risk. But, let's assume that the system behavior will be shown with the $d p$-value equal to $2 \%$ in comparison with the observable window " 1 " (the observable window "2" in Fig. 4a, the middle figure). Then the acting system behavior will exit beyond of the limits for the prospective system behavior. In this example the excess of the potential limits will be about two times (Fig. 4b, combination of the fragments I and II).

Let' consider the second example. Let the prehistory of the system behavior is known within the range of $\alpha$-variation from 38 to 48 (the observable window " 3 " in Fig. 4a, the bottom figure). In this case it is necessary to note that the peculiarity of the $B_{40}$-bifurcation diagram: the branches of the 2-process actually coincide with each other that does not allow to identify formally the 2-process based on Poincaré's periodicity condition in contrast to the previous cases (Fig. 4a, the upper and middle figures). In other words, after the realized bifurcation the image of the bifurcation diagram is distorted too much, that the number of branches remains equal to one and looks like the 1process. So, a qualitative leap of system state can be indistinguishable by experimental way [11]. Let's assume that the system behavior will be shown with the $d p$-value equal to $40 \%$ in comparison with the observable window " 3 " (the observable window " 4 " 
in Fig. 4a, the upper figure). Then the acting system behavior not only will exit essentially beyond of the limits for the prospective system behavior, but also unpredictable dual features will be observed. (Fig. 4c, combination of the fragments III and IV).

The diagrams sewing together pieces of several $B$-bifurcation diagrams are used in the both examples to illustrate the variants of potential limits of the risk. The similar bifurcation diagrams will be hereafter called the $P W$-bifurcation diagram ("Piece-Wise"-bifurcation diagram). These diagrams can be built for one or several bifurcation realizations. The $P W$-bifurcation diagram allows to show up and to classify the potential combinations of risk phenomena with the purpose to estimate more valid the variety of prospective system behavior. So, it becomes possible to discern the system behavior beyond the limits known under the conditions, when nonlinear dynamics is excluded from consideration.

\section{CONCLUSION}

Three modified bifurcation diagrams $(B-, J-, P W-$ bifurcation diagrams) are considered through developing the fractal approach to forming of modified bifurcation diagrams. The "Branch"bifurcation diagrams (B-bifurcation diagram) are intended for the growth in the capacity and adequacy of the preliminary information about nonlinear dynamics regularities. It seems to be important in applications to the methods of real-time nonlinear dynamics identification and forecasting. The "Joint"-bifurcation diagrams ( $J$-bifurcation diagram) are intended to show up and to analyse the uncertainty zones near bifurcation boundaries. It can be used for more valid estimating a stability margin that seems to be important in applications to methods of control system design and methods of adaptation. The "Piece-wise"-bifurcation diagrams ( $P W$-bifurcation diagram) are intended to show up and to classify the potential combinations of risk phenomena taking into account nonlinear behavior. It seems to be important in applications to more valid estimate the ranges of prospective dynamics of the considered system.

\section{REFERENCES}

[1] G.M. Buiatti, J.A. Martin-Ramos, C.H. Rojas Garsia, A.M.R. Amaral, A.J.Marques Cardoso, An online and noninvasive technique for the condition monitoring of capacitors in boost converters, IEEE Trans. on Instrum. and Measur., (59) (2010), pp. 2134-2143.

[2] Yu.V. Kolokolov, S.L. Koschinsky, V.V. Bagrov, Experimental dynamics of electromechanical pulse energy conversion system, IEEE Trans. on Instrum. and Measur., (55) (2006), pp. 35-43.

[3] M.I. Feigin, M.A. Kagan, Emergencies as a manifestation of the effect of bifurcation memory in controlled unstable systems, Int. $J$. of Bifurcation and Chaos, (14), (2004), pp.2439-2447.

[4] W. Wang-Kit Thong, J. Chen, Complex dynamical behaviors of deflection routing on grid networks, Int. J. of Bifurcation and Chaos, (22) 7 (2012), pp.1250169-1 - 1250169-9.

[5] H. Wang, K. He, B. Li, J. Leu, On some recent advances in complex software networks: modeling, analysis, evolution and applications. Int. J. of Bifurcation and Chaos, (22) 2 (2012), pp.1250024-1 - 1250024-15.

[6] N. Marwan, G. Beller, D. Felsenberg, P. Saparin, J. Kurths, Quantifying changes in the spatial structure of trabecular bone, Int. J. of Bifurcation and Chaos, (22) 2 (2012), pp. 1250027-1 - 1250027-12.

[7] A. Vidal, J.-P. Francoise, Canard cycles in global dynamics, Int. J. of Bifurcation and Chaos, (22) 2 (2012), pp.1250026-1 1250026-13.

[8] A. Trevisan, L. Palatella, Chaos and weather forecasting: The role of the unstable subspace in predictability and state estimation problems, Reviews. Int. J. of Bifurcation and Chaos, (2) 12 (2011), pp. 3389-3415.

[9] J. Demongeot, J. Gaudart, A. Lontos, J. Mintsa, E. Promayon, M. Rachdi, Zero-diffusion domains in reaction-diffusion morphogenetic and epidemiologic processes, Int. J. of Bifurcation and Chaos, (22) 2 (2012), pp. 1250028-1 - 1250028-23.

[10] M. Jelali, An overview of control performance assessment technology and industrial applications, Control Engineering Practice, (14) (2006), pp. 441-466.

[11] Yu.V. Kolokolov, A.V. Monovskaya, Estimating the uncertainty of the behavior of a PWM power converter by analyzing a set of experimental bifurcation diagrams, Int. J. of Bifurcation and Chaos, (23) 4 (2013), pp. 1350063-1- 135006314.

[12] M.H. Jensen, P. Bak, T. Bohr, Transition to chaos by interaction of resonances in dissipative systems, I. Circle maps, Physical Rev. A, (30), 1984, pp. 1960-1969.

[13] Yu.V. Kolokolov, A.V. Monovskaya, Fractal principles of multidimensional data structurization for real-time pulse system dynamics forecasting and identification, Chaos, Solitons \& Fractals, (25) 5 (2005), pp. 9911006.

[14] Yu.V. Kolokolov, A.V. Monovskaya, 
Detection of incipient bifurcation phenomenon in pulse converter dynamics, Russian electrical engineering, (84) 1 (2013), pp. 38-43.

[15] Yu.V. Kolokolov, A.V. Monovskaya, To stability margin tuning based on trial actions in relation to PWM converters, Russian electrical engineering, (84) 3 (2013), pp. 15-21.

[16] S. Banerjee, G. Verghese, Nonlinear phenomena in power electronics: attractors, bifurcations, chaos and nonlinear control, IEEE Press, New York, 2001, 441 p.

[17] B. Lehman, R.M. Bass, Switching frequency dependent averaged models for PWM DC-DC converters, IEEE Trans. on Power Electron., (11) (1996), pp. 89-98.

[18] J. Sun, Small-signal methods for AC distributed power systems - a review, IEEE Trans. on Power Electron., (24) (2009), pp. 2545-2554.

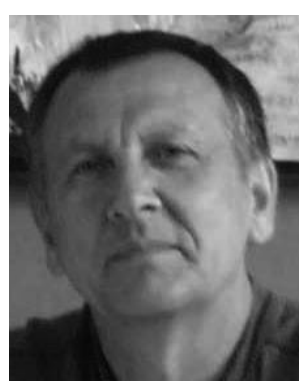

Yury Kolokolov received the M.S. degree in 1972 (Tomsk Politechnical Institute, Russia), PhD degree in 1978 (Tomsk Politechnical Institute, Russia), Russian academic degree of Senior Doctor in 1990 (Tomsk University of Control and Radioelectronics, Russia). From 1993 to 2007 he worked as a Professor and Head of the Dept. of Design and technology of electronic systems (State Technical University of Orel, Russia), from 2007 to 2012 he worked as a Professor and Director of Institute of Control Systems and Information Technologies (UGRA State University, Khanty-Mansiysk, Russia), from 2012 he is a chief researcher (Russian Research Institute of Hydrometeorological Information - World Data Center, Obinsk, Russia). His research interests include theories and practice of control and stability, nonlinear dynamics and catastrophes regarding complex technical, natural and social systems.

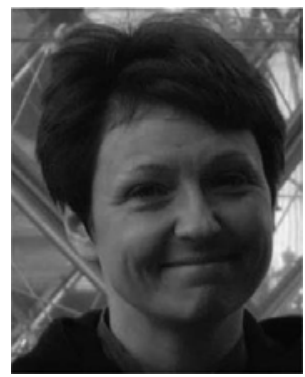

Anna Monovskaya received the M.S. degree in 2001 (State Technical University of Orel, Russia), PhD degree in 2005 (State Technical University of Orel, Russia and University of Technology of Troyes, France), Russian academic degree of Senior Doctor in 2010 (UGRA State University, Khanty-Mansiysk, Russia). From 2007 to 2012 she worked as a Head of the Dept. of Automated systems of data processing and control (UGRA State University, Khanty-Mansiysk, Russia). She is currently a chief researcher (Russian Research Institute of Hydrometeorological Information - World Data Center, Obinsk, Russia). Her research interests include theories and practice of control and stability, nonlinear dynamics and catastrophes regarding complex technical, natural and social systems. 